\title{
Role of SIRT7 in hepatic lipid metabolism
}

\author{
Kazuya Yamagata ${ }^{1}$ Mohammed Fazlul Karim $^{1} \cdot$ Yoshifumi Sato $^{1}$ • \\ Tatsuya Yoshizawa ${ }^{1}$
}

Received: 12 July 2015/Published online: 26 July 2015

(C) The Japan Diabetes Society 2015

Keywords Sirtuin $\cdot$ SIRT7 $\cdot$ Fatty liver $\cdot$ TR4 $\cdot$ E3 ubiquitin ligase complex

Sirtuins are evolutionarily conserved enzymes that regulate a wide variety of biological processes, such as aging, genomic stability, tumorigenesis, and metabolism [1]. To date, seven sirtuins have been identified in mammals (SIRT1-SIRT7), which share a highly conserved NAD ${ }^{+}$ binding and catalytic core domain, but have distinct flanking $\mathrm{N}$ - and $\mathrm{C}$-terminal domains. The divergent $\mathrm{N}$ - and C-termini of sirtuins are responsible for the different functions and subcellular localizations of these enzymes. Although sirtuins were originally described as $\mathrm{NAD}^{+}$-dependent histone deacetylases, these enzymes are now known to act not only on histones, but also on numerous transcription factors and enzymes. SIRT1 controls the acetylation of proliferator-activated receptor- $\gamma$ co-activator $1 \alpha(\mathrm{PGC} 1 \alpha)$, p53, and forkhead box $\mathrm{O}$, among other targets, whereas SIRT3 is a major deacetylase in mitochondria [2, 3]. In addition, a few sirtuins have either weak or undetectable deacetylase activity. For example, SIRT4 is reported to act as an ADP-ribosyltransferase [4], and SIRT5 has both demalonylase and desuccinylase activities [5]. SIRT6 has been shown to deacetylate acetylated lysine 9 of histone H3 (H3K9Ac) and was also recently reported to preferentially hydrolyze long-chain fatty acyl groups on acylated protein targets [6].

Kazuya Yamagata

k-yamaga@kumamoto-u.ac.jp

1 Department of Medical Biochemistry, Faculty of Life Sciences, Kumamoto University, 1-1-1 Honjo, Kumamoto, Kumamoto 860-8556, Japan
SIRT7 mRNA is ubiquitously expressed in all tissues examined to date, with the exception of skeletal muscle [7]. Human (NP_057622.1), mouse (NP_694696.2), and rat (NP_001100543.1) SIRT7 proteins consist of 400, 402, and 402 amino acids, respectively. Human SIRT7 contains a conserved $\mathrm{NAD}^{+}$-binding and catalytic core domain (amino acids 90-331) as well as flanking N-terminal (amino acids 1-89) and C-terminal (332-400) regions (Fig. 1a). The histidine residue at position 187 (H187) (corresponding to mouse H188) is highly conserved among sirtuins and is reported to be important for binding with $\mathrm{NAD}^{+}$[8]. The $\mathrm{N}$-terminal region of SIRT7 contains a nuclear localization signal (NLS) (LQGRSRRREGLKRRQE, amino acids 61-76), and the C-terminal region contains a nucleolar localization signal (NoLS) (KRTKRKKVT, amino acids 392-400) [9]. SIRT7 is enriched in the nucleolus, but is also present in the nucleoplasm (Fig. 1b).

In contrast to SIRT1 to SIRT6, the enzymatic activity and physiological functions of SIRT7 were poorly defined until recently. SIRT7 was first reported to promote ribosomal RNA transcription by interacting with RNA polymerase I (Pol I) and the transcription factor UBF [7, 10]. PAF53, a subunit of Pol I that is required for rDNA transcription, was recently identified as a target of SIRT7 [11]. SIRT7 was also shown to function as $\mathrm{NAD}^{+}$-dependent deacetylase with high selectivity for acetylated lysine 18 of histone H3 (H3K18Ac) [12]. This deacetylase activity plays a role in the gene-specific transcriptional repression of a select subset of H3K18Ac-containing promoters, such as RPS2O and NME1 [12]. H3K18Ac-specific deacetylation by SIRT7 is important for maintaining the phenotype and stabilizing the tumorigenicity of human cancer cells [12]. Our group also demonstrated that Myb-binding protein 1a (Mybbp1a) binds to SIRT7, thereby inhibiting the deacetylation of H3K18 [13]. 
Fig. 1 a Amino acid sequence of human SIRT7 (UniProtKB/ Swiss-Prot: Q9NRC8). The catalytic domain of SIRT7 is shown in red. The highly conserved histidine residue among sirtuins is highlighted in yellow. The $\mathrm{N}$-terminal nuclear localization signal (LQGRSRRREGLKRRQE, amino acids 61-76) and C-terminal nucleolar localization signal (KRTKRKKVT, amino acids 392-400) are indicated by broken boxes. b Intracellular localization of FLAG-tagged SIRT7 in HeLa cells. SIRT7 signals (FLAG staining, green) were detected in the nucleoplasm and nucleoli a

$10 \quad 20 \quad 30 \quad 40$

50

MAAGGLSRSE RKAAERVRRL REEQQRERLR QVSRILRKAA AERSAEEGRL $60 \quad 70 \quad 80 \quad 90 \quad 100$

LAESADLVTE LQGRSRRREG LKRRQEVVD DPEELRGKVR ELASAVRNAK $110 \quad 130 \quad 140 \quad 150$

YLVVYTGAGI STAASIPDYR GPNGVWTLLQ KGRSVSAADL SEAEPTLTHM

$160 \quad 170 \quad 180 \quad 190 \quad 200$

SITRLHEQKL VQHVVSQNCD GLHLRSGLPR TAISELHGNM YIEVCTSCVP

$210 \quad 220 \quad 230 \quad 240 \quad 250$

NREYVRVFDV TERTALHRHQ TGRTCHKCGT QLRDTIVHFG ERGTLGQPLN

$260 \quad 270 \quad 280 \quad 290 \quad 300$

WEAATEAASR ADTILCLGSS LKVLKKYPRL WCMTKPPSRR PKLYIVNLQW

$\begin{array}{lllll}310 & 320 & 330 & 340 & 350\end{array}$

TPKDDWAALK LHGKCDDVMR LLMAELGLEI PAYSRWQDPI FSLATPLRAG

$360370 \quad 380 \quad 390 \quad 400$

EEGSHSRKSL CRSREEAPPG DRGAPLSSAP ILGGWFGRGC TKRTKRKKVT

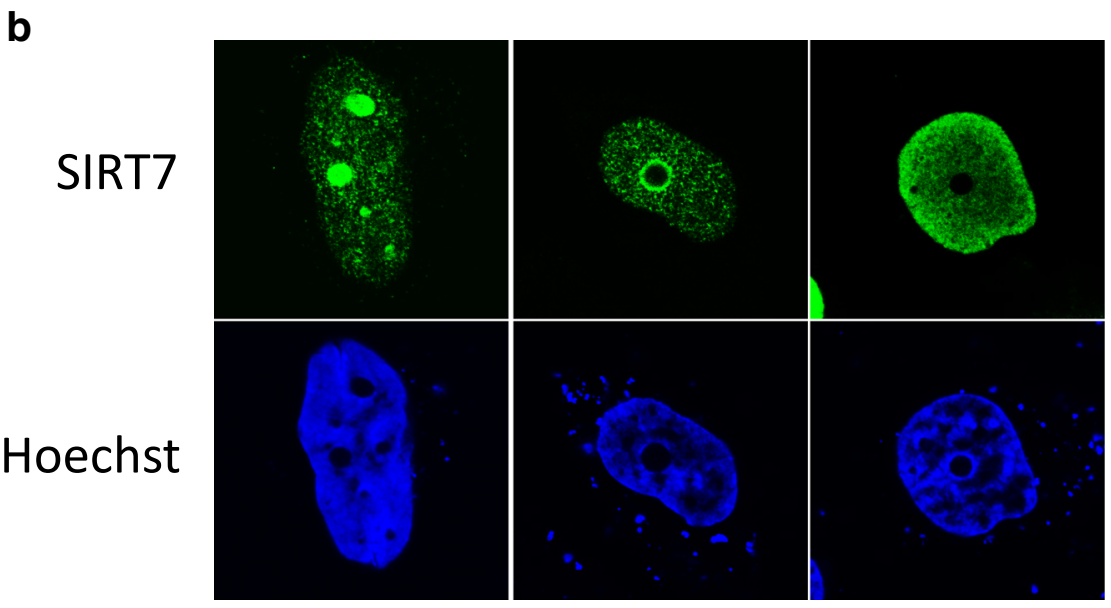

Despite the recent characterization of the binding properties of SIRT7, the metabolic function of SIRT7 has remained largely unknown. As SIRT1, SIRT3, and SIRT6 play critical roles in metabolism, and because the loss of these sirtuins in mice results in the hepatic accumulation of lipids [14-16], we examined whether the loss of SIRT7 affected lipid metabolism in mice. In striking contrast to Sirt1, Sirt3, and Sirt6 knockout (KO) mice, Sirt7 KO mice fed a high-fat diet (HFD) showed less accumulation of hepatic lipid than wild-type (WT) controls [17]. Hepatic triglyceride accumulation was also attenuated in liverspecific Sirt7 KO mice fed an HFD.

The nuclear receptor TR4 regulates the expression of $C d 36$, cell death-inducing DFFA-like effector a (Cidea), cell death-inducing DFFA-like effector c ( $\mathrm{Cidec}$ ), monoacylglycerol $O$-acyltransferase 1 (Mogat1), and peroxisome proliferator activated receptor gamma (Pparg), and it is a key factor in the regulation of lipid homeostasis [18]. Among these proteins, CD36 plays a crucial role in fatty acid uptake [19], and CIDE-A and CIDE-C are involved in lipid storage, lipid droplet formation, and lipolysis [20]. In addition, MOGAT1 catalyzes the synthesis of diacylglycerol, the precursor of triglyceride (TG). Thus, the activation of hepatic TR4 increases fatty acid uptake and TG synthesis/storage in the liver. Notably, the hepatic level of TR4 protein is decreased in Sirt7 KO mice as well as in several types of hepatic cells with Sirt7 knockdown (KD) using siRNA [17]. In addition, the overexpression of WT-SIRT7, but not inactive SIRT7-H188Y mutant protein, restores TR4 expression in Sirt7 KD AML-12 mouse hepatocyte cells, suggesting that the $\mathrm{NAD}^{+}$-dependent enzymatic activity of SIRT7 is required for the regulation of TR4 expression [17]. Furthermore, the observed decrease of hepatic lipid accumulation and $C d 36$ expression in Sirt7 
$\mathrm{KO}$ mice is restored to control levels by infection with TR4-expressing adenovirus. Taken together, these results suggest that hepatic SIRT7 controls hepatic lipid accumulation and functions, at least partly, in a TR4-dependent manner.

Our group has also demonstrated that TR4 mRNA levels are unchanged in the livers of Sirt7 KO mice and in Sirt7 KD AML-12 cells. Mechanistically, we found that SIRT7 binds to and prevents the DCAF1/DDB1/CUL4B E3 ubiquitin ligase complex from degrading TR4 [17]. Therefore, the decreased accumulation of hepatic lipids in Sirt7 KO mice may be attributable to the decreased levels of TR4 due to the increased activity of the E3 ubiquitin ligase complex (Fig. 2). To our knowledge, this is the first example of the functional regulation of a ubiquitin ligase complex by the direct binding of a sirtuin. However, the exact molecular mechanism of how SIRT7 inhibits the function of the DCAF1/DDB1/CUL4B complex remains unclear.

Two other research groups have also independently generated and characterized the hepatic phenotypes of Sirt7
KO mice. Shin et al. reported that SIRT7 is induced by endoplasmic reticulum (ER) stress and is stabilized in the promoter regions of genes encoding ribosomal proteins through interaction with Myc, resulting in the silencing of ribosomal protein gene expression [21]. In their study, Sirt7 KO mice fed a chow diet develop fatty liver because of reduced suppression of ER stress [21]. Notably, however, the expression of ER stress-related genes was not increased in livers of our Sirt7 KO mice (unpublished data) or another line of Sirt7-deficient mice [22]. GA-binding protein $(\mathrm{GABP})$ is a nuclear transcription factor that is important for mitochondrial biogenesis and function. The GABP complex is composed of two subunit proteins, $\mathrm{GABP} \alpha$ and GABP $\beta 1$, which function in DNA binding and transcriptional activation, respectively. Ryu et al. found that SIRT7 deacetylates GABP $\beta 1$, facilitating its transcriptional activation through complex formation with GABP $\alpha$. They also reported that aged (42 weeks old) Sirt7 $\mathrm{KO}$ mice exhibit hepatic microvesicular steatosis because of mitochondrial dysfunction [22]. As we mainly examined young (11-17 weeks of age) Sirt7 KO mice fed an HFD, it

\section{$\operatorname{SIRT7}(+/+)$}

Decreased activity of E3 ubiquitin ligase complex

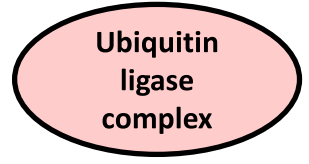

Increased expression of TR4 transcription factor

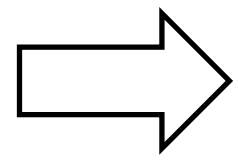

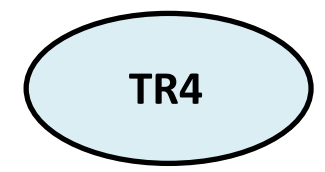

Increased expression of $C d 36$, Cidea, Cidec, Mogat1, Pparg mRNAs

\section{arg}

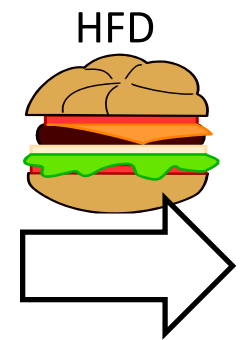

\section{SIRT7(-/-)}

Increased activity of E3 ubiquitin ligase complex

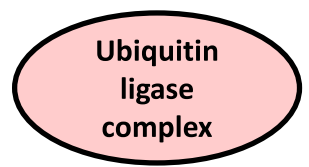

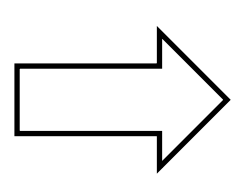

Decreased expression of TR4 transcription factor

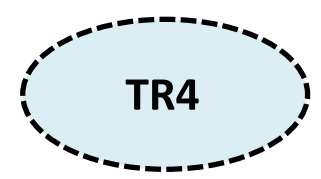

Decreased expression of

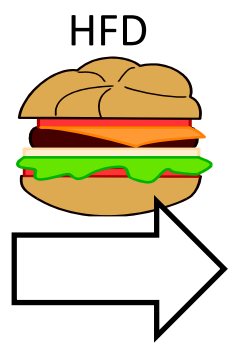

cd36, Cidea, Cidec, Mogat1, Pparg mRNAs

\section{Fatty liver}

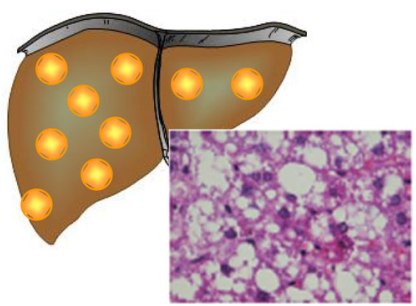

Improvement of fatty liver

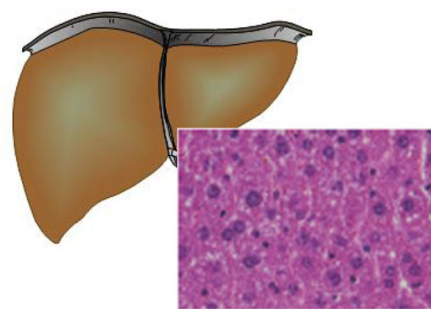

Fig. 2 Schematic representation of SIRT7-mediated regulation of TR4-dependent hepatic lipid metabolism 
is necessary to investigate hepatic lipid accumulation in aged Sirt7 KO mice. Although we have no adequate explanation (e.g., targeting construct, genetic background, or gut microbiota) for these different phenotypes at present, these studies demonstrate that SIRT7 has important roles in hepatic lipid metabolism. Further studies are necessary to improve our understanding of the function of SIRT7 in humans.

\section{Compliance with ethical standards}

Conflict of interest The authors declare that there is no conflict of interest.

Human rights statement and informed consent This article does not contain any studies with human or animal subjects performed by the any of the authors.

\section{References}

1. Guarenete L. Sir2 links chromatin silencing, metabolism, and aging. Genes Dev. 2000;14:1201-6.

2. Houtkooper RH, Pirinen E, Auwerx J. Sirtuins as regulators of metabolism and healthspan. Nat Rev Mol Cell Biol. 2012;13:225-38.

3. Zhong L, Mostoslavsky R. Fine tuning our cellular factories: sirtuins in mitochondrial biology. Cell Metab. 2011;13:621-6.

4. Haigis MC, Mostoslavsky R, Haigis KM, Fahie K, Christodoulou DC, Murphy AJ, Valenzuela DM, Yancopoulos GD, Karow M, Blander G, Wolberger C, Prolla TA, Weindruch R, Alt FW, Guarente L. SIRT4 inhibits glutamate dehydrogenase and opposes the effects of calorie restriction in pancreatic beta cells. Cell. 2006;126:941-54.

5. Du J, Zhou Y, Su X, Yu J, Khan S, Jiang H, Kim J, Woo J, Kim $\mathrm{JH}$, Choi BH, et al. Sirt5 is a NAD-dependent protein lysine demalonylase and desuccinylase. Science. 2011;334:806-9.

6. Jiang H, Khan S, Wang Y, Charron G, He B, Sebastian C, Du J, Kim R, Ge E, Mostoslavsky R, et al. SIRT6 regulates TNF- $\alpha$ secretion through hydrolysis of long-chain fatty acyl lysine. Nature. 2013;496:110-3.

7. Ford E, Voit R, Liszt G, Magin C, Grummt I, Guarente L. Mammalian Sir2 homolog SIRT7 is an activator of RNA polymerase I transcription. Genes Dev. 2006;20:1075-80.

8. Finnin MS, Donigian JR, Pavletich NP. Structure of the histone deacetylase SIRT2. Nat Struct Biol. 2001;8:621-5.

9. Kiran S, Chatterjee N, Singh S, Kaul SC, Wadhwa R, Ramakrishna G. Intracellular distribution of human SIRT7 and mapping of the nuclear/nucleolar localization signal. FEBS J. 2013;280:3451-66.

10. Grob A, Roussel P, Wright JE, McStay B, Hernandez-Verdun D, Sirri V. Involvement of SIRT7 in resumption of rDNA transcription at the exit from mitosis. J Cell Sci. 2009;122:489-98.
11. Chen S, Seiler J, Santiago-Reichelt M, Felbel K, Grummt I, Voit R. Repression of RNA polymerase I upon stress is caused by inhibition of RNA-dependent deacetylation of PAF53 by SIRT7. Mol Cell. 2013;52:303-13.

12. Barber MF, Michishita-Kioi E, Xi Y, Tasselli L, Kioi M, Moqtaderi Z, Tennen RI, Paredes S, Young NL, Chen K, et al. SIRT7 links H3K18 deacetylation to maintenance of oncogenic transformation. Nature. 2012;487:114-8.

13. Karim MF, Yoshizawa T, Sato Y, Sawa T, Tomizawa K, Akaike T, Yamagata K. Inhibition of H3K18 deacetylation of Sirt7 by Myb-binding protein 1a (Mybbp1a). Biochem Biophys Res Commun. 2013;441:157-63.

14. Purushotham A, Schug TT, Xu Q, Surapureddi S, Guo X, Li X. Hepatocyte-specific deletion of SIRT1 alters fatty acid metabolism and results in hepatic steatosis and inflammation. Cell Metab. 2009;9:327-38.

15. Hirschey MD, Shimazu T, Goetzman E, Jing E, Lombard DB, Grueter CA, Harris C, Biddinger S, Ilkayeva OR, Stevens RD, et al. SIRT3 regulates fatty acid oxidation via reversible enzyme deacetylation. Nature. 2010;464:121-5.

16. Kim HS, Xiao C, Wang RH, Lahusen T, Xu X, Vassilopoulos A, Vazquez-Ortiz G, Jeong WI, Park O, Ki SH, et al. Hepatic specific disruption of SIRT6 in mice results in fatty liver formation due to enhanced glycolysis and triglyceride synthesis. Cell Metab. 2010;12:224-36.

17. Yoshizawa T, Karim MF, Sato Y, Senokuchi T, Miyata K, Fukuda T, Go C, Tasaki M, Uchimura K, Kadomatsu T, Tian Z, Smolka C, Sawa T, Takeya M, Tomizawa K, Ando Y, Araki E, Akaike T, Braun T, Oike Y, Bober E, Yamagata K. SIRT7 controls hepatic lipid metabolism by regulating the ubiquitinproteasome pathway. Cell Metab. 2014;19:712-21.

18. Kang HS, Okamoto K, Kim Y-S, Takeda Y, Bortner CD, Dang H, Wada T, Xie W, Yang X-P, Liao G, et al. Nuclear orphan receptor TAK1/TR4-deficient mice are protected against obesitylinked inflammation, hepatic steatosis, and insulin resistance. Diabetes. 2011;60:177-88.

19. Koonen D, Jacobs R, Febbraio M, Young ME, Soltys C-LM, Ong $\mathrm{H}$, Vance DE, Dyck JR. Increased hepatic CD36 expression contributes to dyslipidemia associated with diet-induced obesity. Diabetes. 2007;56:2863-71.

20. Matsusue K, Kusakabe T, Noguchi T, Takiguchi S, Suzuki T, Yamano S, Gonzalez FJ. Hepatic steatosis in leptin-deficient mice is promoted by the PPARgamma target gene Fsp27. Cell Metab. 2008;7:302-11.

21. Shin J, He M, Liu Y, Paredes S, Villanova L, Brown K, Qiu X, Nabavi N, Mohrin M, Wojnoonski K, Li P, Cheng HL, Murphy AJ, Valenzuela DM, Luo H, Kapahi P, Krauss R, Mostoslavsky R, Yancopoulos GD, Alt FW, Chua KF, Chen D. SIRT7 represses Myc activity to suppress ER stress and prevent fatty liver disease. Cell Rep. 2013;5:654-65.

22. Ryu D, Jo YS. Lo Sasso G, Stein S, Zhang H, Perino A, Lee JU, Zeviani M, Romand R, Hottiger MO, Schoonjans K, Auwerx J. A SIRT7-dependent acetylation switch of GABP $\beta 1$ controls mitochondrial function. Cell Metab. 2014;20:856-69. 